\title{
Analytical Expression for Impulse Response Between Two Nodes in 2-D Rectangular Digital Waveguide Mesh
}

\author{
Zhixin Chen, Student Member, IEEE, and Robert C. Maher, Senior Member, IEEE
}

\begin{abstract}
The digital waveguide mesh models multidimensional wave propagation in discrete space and time. The finite difference wave equation used in the mesh is expressed in the space-time domain, which is not straightforward to reveal the analytical expression for the impulse response between a source and a receiver. This letter describes a procedure to derive an analytical impulse response expression between two nodes in a 2 -D rectangular digital waveguide mesh based on the wave equation. The analytical expression can provide helpful insights and help verify simulation results for the digital waveguide mesh.
\end{abstract}

Index Terms-Acoustic propagation, digital waveguide mesh, impulse response expression, multidimensional signal processing, room acoustics modeling.

\section{INTRODUCTION}

$\mathbf{T}$ HE DIGITAL waveguide mesh provides a numerical solution to the wave equation in multiple dimensions and, thus, has the benefit of incorporating the effects of diffraction and wave interference [1]-[3]. The 2-D and 3-D digital waveguide mesh schemes have been used to simulate wave propagation in musical instruments [4]-[6] and acoustic spaces [7], [8].

The 2-D rectangular mesh can suffer from frequency and direction dependent dispersion. The error can be reduced by using an interpolated mesh structure [9] or a triangular mesh topology [4], [10], often in combination with a frequency warping technique [9]. The interpolated mesh structure and the triangular mesh topology improve the performance of the mesh by modifying the finite difference expression of the wave equation. However, the modified schemes of the wave equation are still in the space-time domain, and it is not straightforward to find an analytical expression for the impulse response between a source and a receiver in the mesh. The procedure described in this letter derives an analytical expression for the impulse response between two nodes in a 2-D rectangular mesh and verifies the simulation results of the mesh.

The remaining sections of this letter are organized as follows. The basic topology of the 2-D rectangular mesh is briefly reviewed in Section II. The work deriving the analytical expres-

Manuscript received July 20, 2007; revised November 26, 2007. This work was supported by a Benjamin Ph.D. Fellowship from Montana State University. The associate editor coordinating the review of this manuscript and approving it for publication was Dr. Patrick A. Naylor.

The authors are with the Department of Electrical and Computer Engineering, Montana State University, Bozeman, MT 59717-3780 USA (e-mail: chen.zhixin.mt@gmail.com; rob.maher@montana.edu).

Digital Object Identifier 10.1109/LSP.2007.916043

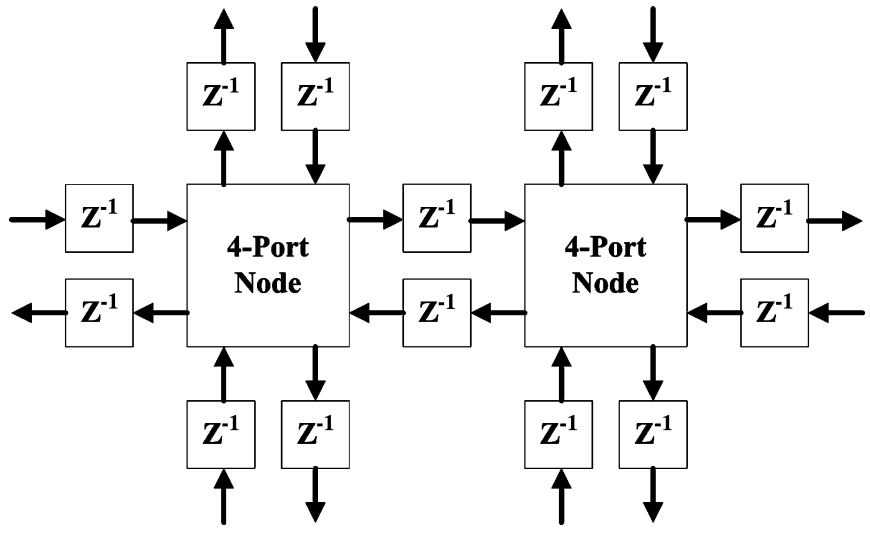

Fig. 1. In the 2-D rectangular digital waveguide mesh, each node is connected to four neighbors with unit delays.

sion of the impulse response between two nodes in a 2-D rectangular mesh is described in Section III. The simulation results of the impulse responses for three arbitrary source-receiver positions using the mesh and the derived expression are compared in Section IV. This letter is concluded in Section V.

\section{The 2-D Rectangular Digital WaVeguide Mesh}

The rectangular digital waveguide mesh is a regular array of 1-D digital waveguides arranged along each perpendicular dimension, interconnected at their crossings by scattering nodes with unit delay elements. A 2-D rectangular digital waveguide mesh was originally proposed in [1] and is illustrated in Fig. 1.

The difference wave equation for 2-D rectangular mesh can be expressed using the node values as

$v(n, i, j)=\frac{1}{2}[v(n-1, i+1, j)+v(n-1, i-1, j)$
$+v(n-1, i, j+1)+v(n-1, i, j-1)]-v(n-2, i, j)$

where $v$ represents the wave velocity signal of a node at rectangular coordinates $(i, j)$ at time index $n$. Here $i, j$, and $n$ are arbitrary integers.

\section{Impulse Response BetweEn Two Nodes IN 2-D RECTANGULAR MESH}

When a 2-D rectangular mesh is used for simulations, one frequently asked question is how to obtain an expression for the impulse response between a source $S$ at node $(A, B)$ and 


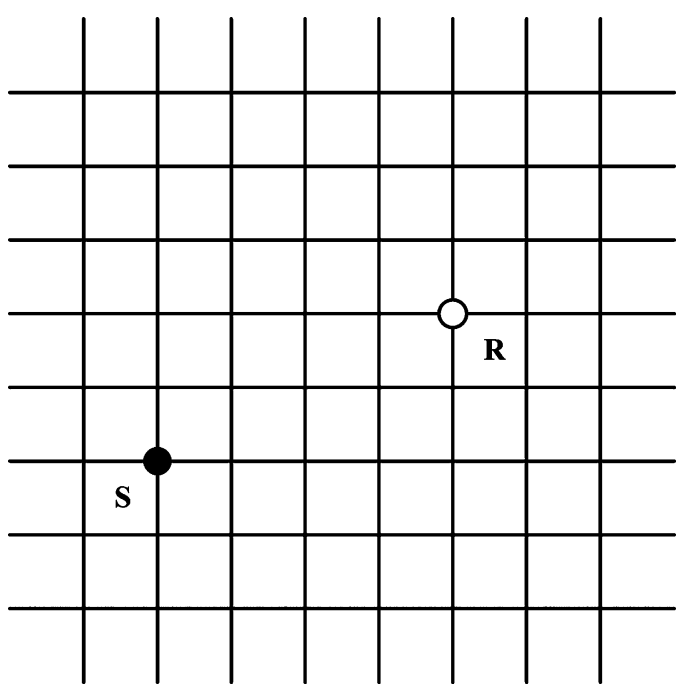

Fig. 2. An example source node $\mathrm{S}$ and an example receiver node $\mathrm{R}$ are located in a 2-D rectangular digital waveguide mesh.

a receiver $R$ at node $(A+M, B+N)$, i.e., how to find an expression for the wave velocity signal at the receiver node $(A+$ $M, B+N)$ if an impulse signal is fed into the source node $(A, B)$ at time index $n=0$.

The impulse response question can also be mathematically expressed as follows. Given a time-space impulse signal $\delta(n, i-$ $A, j-B)$, what will be the analytical expression of the received wave velocity signal $v(n, A+M, B+N)$ for $n=0,1,2,3, \cdots$ ? Here the time-space impulse signal $\delta(n, i-A, j-B)$ is defined as follows:

$$
\delta(n, i-A, j-B)= \begin{cases}1, & n=0, i=A, j=B \\ 0, & \text { otherwise }\end{cases}
$$

The impulse response can certainly be simulated directly using (1), but an analytical expression of the impulse response can provide helpful insights and help verify the simulation results.

In an unbounded rectangular mesh, $v(n, A+M, B+N)$ is equal to $v(n, A+|M|, B+|N|)$ due to the symmetry of propagation in front of a time-space impulse signal $\delta(n, i-A, j-B)$. Thus, without loss of generality, both $M$ and $N$ are assumed to be nonnegative integers here. An example source-receiver layout is shown in Fig. 2.

It should be noticed that given an initial excitation at some node in the mesh, the excitation energy will spread out from the excitation node more and more as the traveling waves scatter through the nodes. This phenomenon can be described using 2-D convolution [11]. To facilitate the analysis of the impulse response question, one 2-D spatial signal $h$ is defined as follows:

$$
\begin{aligned}
h & =\left\{h\left(m_{1}, m_{2}\right),-\infty<m_{1}, m_{2}<\infty\right\} \\
& = \begin{cases}0.5, & m_{1}=A \pm 1, m_{2}=B \pm 1 \\
0, & \text { otherwise }\end{cases}
\end{aligned}
$$

According to (1), if an impulse signal is fed into the source node $(A, B)$ at time index $n=0$, the evolution of the wave velocity signal in the nodes over time can be expressed as

$$
v(n, i, j)=h * * v(n-1, i, j)-v(n-2, i, j)
$$

where "**" denotes 2-D convolution and $v(n, i, j)=\delta(n, i-$ $A, j-B)$ for $n \leq 0$. Here the wave velocity signal $v(n, i, j)$ is viewed as a 2-D spatial signal for specific time index $n$.

Given $v(n, i, j)=\delta(n, i-A, j-B)$ for $n \leq 0$, using (2) iteratively, the wave velocity $v(n, i, j)$ can be written as

$$
v(n, i, j)=\sum_{t=0}^{\left[\frac{n}{2}\right]}(-1)^{t} C_{n-t}^{t} h^{(n-2 t) * *}, \quad \text { for } n \geq 1
$$

where $[n / 2]$ means the largest integer value less than or equal to $n / 2, C_{n-t}^{t} \equiv(n-t) ! / t !(n-2 t) !$ is the binomial coefficient, and $h^{(n-2 t) * *} \equiv \underbrace{h * * h \cdots h * * h}_{n-2 t}$ means the $n-2 t$ fold 2-D convolution of $h$ with itself. The calculation of wave velocity signal $v(n, i, j)$ thus needs to obtain the value of the polynomials obtained by self-convolving $h$ for $n-2 t$ times. Specifically, calculating the received wave velocity $v(n, A+M, B+N)$ requires computing what the $h$ polynomials equal at the receiver node $(A+M, B+N)$.

If an impulse signal is fed into the source node $(A, B)$ at time index $n=0$ and continuously convolved with the 2-D signal $h$, the evolution of the node value over time becomes $h^{n * *}$ at time index $n$. Therefore, given $\hat{v}(n, i, j)=\delta(n, i-A, j-B)$ for $n \leq 0$, the calculation of $h^{n * *}$ is actually solving $\hat{v}(n, i, j)=$ $h * * \hat{v}(n-1, i, j)$, i.e., solving

$$
\begin{aligned}
\hat{v}(n, i, j)=\frac{1}{2}[ & \hat{v}(n-1, i+1, j)+\hat{v}(n-1, i-1, j) \\
& +\hat{v}(n-1, i, j+1)+\hat{v}(n-1, i, j-1)] .
\end{aligned}
$$

Von Neumann analysis can be applied to the difference equation to show the evolution of the spatial spectrum over time by using Fourier transform theory [1], [12]. To calculate $h^{n * *}$ at the receiver node $(A+M, B+N)$, Von Neumann analysis applied to (4) yields

$$
\begin{aligned}
\hat{V}\left(n, \xi_{1}, \xi_{2}\right)= & \frac{1}{2}\left[e^{j 2 \pi \xi_{1} c T}+e^{-j 2 \pi \xi_{1} c T}\right. \\
& \left.+e^{j 2 \pi \xi_{2} c T}+e^{-j 2 \pi \xi_{2} c T}\right] \hat{V}\left(n-1, \xi_{1}, \xi_{2}\right)
\end{aligned}
$$

where $\hat{V}\left(n, \xi_{1}, \xi_{2}\right)$ is the 2-D discrete-space Fourier transform of $\hat{v}(n, i, j), \xi_{1}$ and $\xi_{2}$ are the spatial frequencies, $c$ is the sound velocity, and $T$ is the temporal sampling interval.

Equation (5) can be represented using a spectral amplification factor, $g\left(\xi_{1}, \xi_{2}\right)$. Multiplying the Fourier transform of the solution by $g\left(\xi_{1}, \xi_{2}\right)$ is equivalent to advancing the solution of the time-domain scheme by one time step [1], [12]. Deriving $g\left(\xi_{1}, \xi_{2}\right)$ from (5) gives

$$
\begin{aligned}
\hat{V}\left(n, \xi_{1}, \xi_{2}\right)= & g^{n}\left(\xi_{1}, \xi_{2}\right) \hat{V}\left(0, \xi_{1}, \xi_{2}\right) \\
= & \frac{1}{2}\left[e^{j 2 \pi \xi_{1} c T}+e^{-j 2 \pi \xi_{1} c T}+e^{j 2 \pi \xi_{2} c T}\right. \\
& \left.\quad+e^{-j 2 \pi \xi_{2} c T}\right] g^{n-1}\left(\xi_{1}, \xi_{2}\right) \hat{V}\left(0, \xi_{1}, \xi_{2}\right)
\end{aligned}
$$


where $\hat{V}\left(0, \xi_{1}, \xi_{2}\right)$ represents the initial conditions at time index $n=0$. The amplification factor $g\left(\xi_{1}, \xi_{2}\right)$ is calculated as

$$
\begin{aligned}
g\left(\xi_{1}, \xi_{2}\right) & =\frac{1}{2}\left[e^{j 2 \pi \xi_{1} c T}+e^{-j 2 \pi \xi_{1} c T}+e^{j 2 \pi \xi_{2} c T}+e^{-j 2 \pi \xi_{2} c T}\right] \\
& =\frac{1}{2}\left[e^{j \phi_{1}}+e^{-j \phi_{1}}+e^{j \phi_{2}}+e^{-j \phi_{2}}\right]
\end{aligned}
$$

where $\phi_{1}=2 \pi \xi_{1} c T$ and $\phi_{2}=2 \pi \xi_{2} c T$.

According to the spatial shift theorem of the 2-D Fourier transform, to calculate the value at the receiver node $(A+M, B+N)$, only the coefficient of $e^{j M \phi_{1}} e^{j N \phi_{2}}$ from the polynomial $g^{n}\left(\xi_{1}, \xi_{2}\right)$ needs to be computed. To get the factor $e^{j M \phi_{1}} e^{j N \phi_{2}}$, the difference in the exponents of $e^{j \phi_{1}}$ and $e^{-j \phi_{1}}$ should be equal to $M$ and the difference in the exponents of $e^{j \phi_{2}}$ and $e^{-j \phi_{2}}$ should equal $N$. In the 2-D rectangular mesh, the path length of every route between the source node $(A, B)$ and the receiver node $(A+M, B+N)$ is always an even or an odd number of unit delays, so the coefficient of $e^{j M \phi_{1}} e^{j N \phi_{2}}$ is equal to 0 for every other time step. In addition, the coefficient of $e^{j M \phi_{1}} e^{j N \phi_{2}}$ equals 0 for the time index $n<M+N$ since it takes $M+N$ time steps for the input to propagate from the source to the receiver. Based on these considerations, expanding the bracketed expression in $g^{n}\left(\xi_{1}, \xi_{2}\right)=\left[(1 / 2)\left(e^{j \phi_{1}}+e^{-j \phi_{1}}+e^{j \phi_{2}}+e^{-j \phi_{2}}\right)\right]^{n}$, the factor $e^{j M \phi_{1}} e^{j N \phi_{2}}$ is only included in the polynomial

$$
\begin{aligned}
\frac{1}{2^{n}}[ & C_{n}^{N}\left(e^{j \phi_{1}}+e^{-j \phi_{1}}\right)^{n-N}\left(e^{j \phi_{2}}+e^{-j \phi_{2}}\right)^{N} \\
& +C_{n}^{N+2}\left(e^{j \phi_{1}}+e^{-j \phi_{1}}\right)^{n-N-2}\left(e^{j \phi_{2}}+e^{-j \phi_{2}}\right)^{N+2}+\ldots \\
& \left.+C_{n}^{n-M}\left(e^{j \phi_{1}}+e^{-j \phi_{1}}\right)^{M}\left(e^{j \phi_{2}}+e^{-j \phi_{2}}\right)^{n-M}\right]
\end{aligned}
$$

where $n=M+N, M+N+2, \cdots$. The coefficient of $e^{j M \phi_{1}} e^{j N \phi_{2}}$ in each term is calculated as follows:

$$
\begin{aligned}
\frac{1}{2^{n}}\left(C_{n}^{N} C_{n-N}^{\frac{n-M-N}{2}} C_{N}^{0}+C_{n}^{N+2} C_{n-N-2}^{\frac{n-M-N}{2}-1} C_{N+2}^{1}+\cdots\right. \\
\left.\quad+C_{n}^{n-M} C_{M}^{0} C_{n-M}^{\frac{n-M-N}{2}}\right) \\
=\frac{1}{2^{n}} \sum_{t=0}^{\frac{n-M-N}{2}}\left(C_{n}^{N+2 t} C_{n-N-2 t}^{\frac{n-M-N}{2}-t} C_{N+2 t}^{t}\right) \\
=\frac{1}{2^{n}} \sum_{t=0}^{\frac{n-M-N}{2}}\left(C_{n}^{\frac{n-M-N}{2}} C_{\frac{n-M-N}{2}}^{t} C_{\frac{n+M+N}{2}}^{N+t}\right) \\
=\frac{C_{n}^{\frac{n-M-N}{2}} C_{n}^{\frac{n-M+N}{2}}}{2^{n}} M+N, M+N+2, \cdots .
\end{aligned}
$$

According to the shift theorem of the 2-D Fourier transform, the value of $h^{n * *}$ at the receiver node $(A+M, B+N)$ is expressed as

$$
\begin{aligned}
& \hat{v}(n, A+M, B+N) \\
& \quad= \begin{cases}\frac{C_{n}^{\frac{n-M-N}{2}} C_{n}^{\frac{n-M+N}{2}}}{2^{n}}, & \text { for } n=M+N, M+N+2, \cdots \\
0, & \text { otherwise. }\end{cases}
\end{aligned}
$$

Substituting the expression for $\hat{v}(n, A+M, B+N)$ into (3) gives the wave velocity signal $v(n, A+M, B+N)$, i.e., the impulse response between the source node $(A, B)$ and the receiver node $(A+M, B+N)$, which is expressed in (10) at the bottom of the page.

In (10), the impulse response is analytically expressed as the summation of combined polynomials in the time domain. The computational complexity is high in this equation, so its practical usefulness is limited. However, for a particular case when the receiver node is in the diagonal direction of the source node, i.e., $M=N$, some simplifications can be observed by successively incrementing $n$ in (10).

When $n=2 N, v(2 N, A+N, B+N)=C_{2 N}^{N} / 2^{2 N}$. From the calculation result in (10), observe that an update expression for $n=2 N, 2 N+4,2 N+8 \cdots$ can be written as $v(n+4, A+$ $N, B+N)=v(n, A+N, B+N)((n-2 N+2)(n+2 N+$ $2) /(n-2 N+4)(n+2 N+4))$ and that $v(n, A+N, B+N)=0$ for other values of $n$.

According to these observations, (10) can be further simplified to the following compact form:

$$
\begin{aligned}
v(n, A & +N, B+N) \\
& = \begin{cases}\frac{C_{\frac{n-2 N}{4}}^{\frac{n-2 N}{2}} C^{\frac{n+2 N}{4}}}{2}, & \text { for } n=2 N, 2 N+4, \cdots \\
0, & \text { otherwise. }\end{cases}
\end{aligned}
$$

Note that in (11), every three out of four samples in $v(n, A+$ $N, B+N)$ are zero-valued.

\section{Simulation Results}

The impulse responses for three arbitrary source-receiver positions were simulated using (1) and (10), respectively. The simulations using (1) were made by constructing a $200 \times 200$ node 2-D rectangular mesh and applying an impulse signal into source node $(80,120)$. The impulse responses were then simulated at arbitrary receiver nodes $(80,120),(83,122)$, and $(85,125)$, which correspond to $M=0$ and $N=0, M=3$ and $N=2$, and $M=5$ and $N=5$, respectively. In the simulations, sound velocity was set to be $343.5 \mathrm{~m} / \mathrm{s}$ and spatial sampling interval was set to be $0.0101 \mathrm{~m}$, indicating an updating

$$
v(n, A+M, B+N)= \begin{cases}\frac{n-M-N}{\sum_{t=0}^{2}} \frac{(-1)^{t} C_{n-t}^{t} C_{n-2 t}^{\frac{n-M-N-2 t}{2}} C_{n-2 t}^{\frac{n-M+N-2 t}{2}}}{2^{n-2 t}}, & \text { for } n=M+N, M+N+2, \cdots \\ 0, & \text { otherwise }\end{cases}
$$



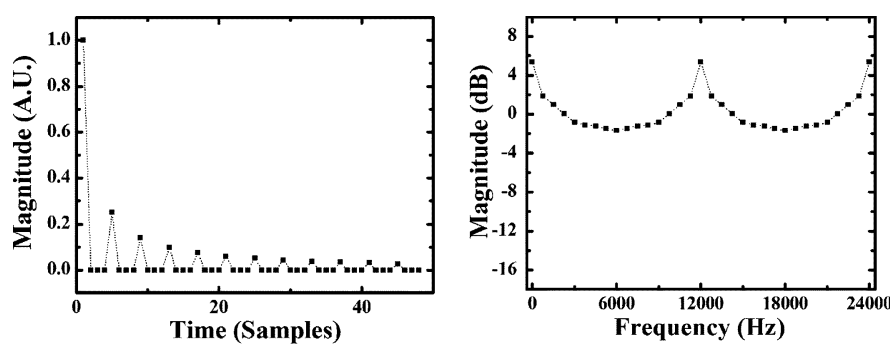

(a)
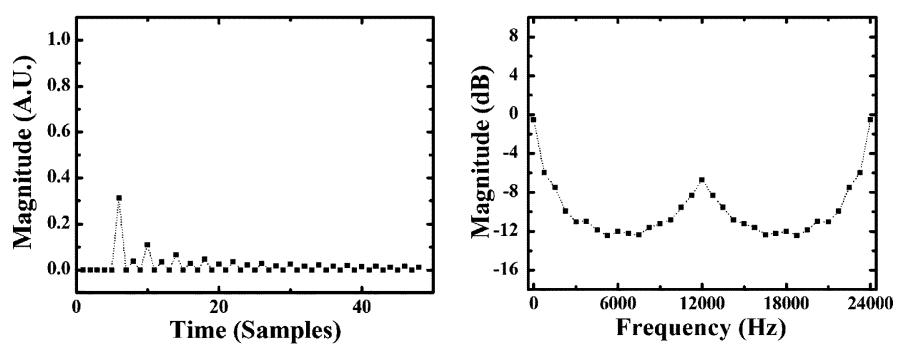

(b)
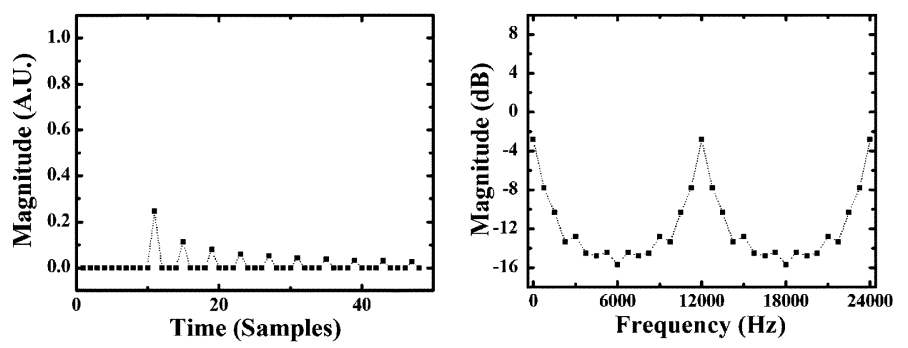

(c)

Fig. 3. Impulse response (left figure) and magnitude frequency response (right figure) for three example source-receiver positions in 2-D rectangular digital waveguide mesh. Here A.U. represents arbitrary unit. (a) $\mathrm{M}=0$ and $\mathrm{N}=0$. (b) $\mathrm{M}=3$ and $\mathrm{N}=2$. (c) $\mathrm{M}=5$ and $\mathrm{N}=5$.

frequency of $48 \mathrm{kHz}$. The impulse response simulations were run for 48 time steps, which ensured that the sound wave would not propagate out of the mesh boundary. The three impulse responses were obtained and also numerically transformed into the corresponding frequency responses using an FFT length of 64 samples. The simulation results for the three source-receiver positions were identical to those using (10). The simulation results for the receiver nodes $(80,120)$ and $(85,125)$ were also identical to those using (11), as expected.

Fig. 3 shows the three impulse responses and the corresponding magnitude frequency responses. In every magnitude frequency response, the spectrum is symmetric about one quarter of the updating frequency. This symmetry characteristic in the frequency domain corresponds to the phenomenon that every other sample is zero-valued in the time domain, as shown in (10) and in the simulated impulse responses in Fig. 3.

\section{CONCLUSION}

The derivation of the analytical expression for the impulse response between two nodes in a 2-D rectangular digital waveguide mesh was described in this letter. A compact time-domain analytical expression for the impulse response was obtained when the receiver was in the diagonal direction of the source. For other source-receiver layouts, the impulse response was analytically expressed as the summation of combined polynomials in the time domain. This derived analytical expression for an impulse response can be used to verify simulation results obtained from a 2-D rectangular digital waveguide mesh and to facilitate better understanding of its associated characteristics.

In this letter, the analytical expression of the impulse response is only derived in the unbounded 2-D rectangular mesh,but the derivation procedure may be used in more complicated problems by modifying or adding some parameters. For example, if the derivation procedure is to be used in unbounded triangular mesh, the 2-D spatial signal $h$ needs to be changed. If the procedure is to be applied in the bounded 2-D rectangular mesh, the expression of the boundary condition should be considered.

\section{REFERENCES}

[1] S. Van Duyne and J. O. Smith, "Physical modeling with the 2-D digital waveguide mesh," in Proc. Int. Computer Music Conf., Tokyo, Japan, Sep. 1993, pp. 40-47.

[2] S. Van Duyne and J. O. Smith, "The 2-D digital waveguide mesh," in Proc. IEEE Workshop Applicat. Signal Process. Audio Acoust., New Paltz, NY, Oct. 1993.

[3] D. T. Murphy, A. Kelloniemi, J. Mullen, and S. Shelley, "Acoustic modeling using the digital waveguide mesh," IEEE Signal Process. Mag., vol. 24, no. 2, pp. 55-66, Mar. 2007.

[4] F. Fontana and D. Rocchesso, "A new formulation of the 2-D waveguide mesh for percussion instruments," in Proc. XI Colloq. Musical Informatics, Bologna, Italy, Nov. 1995, pp. 27-30.

[5] F. Fontana and D. Rocchesso, "Signal-theoretic characterization of waveguide mesh geometries for models of two-dimensional wave propagation in elastic media," IEEE Trans. Speech Audio Process., vol. 9, no. 2, pp. 152-161, Feb. 2001.

[6] M. Aird, J. Laird, and J. Fitch, "Modelling a drum by interfacing 2-D and 3-D waveguide meshes," in Proc. Int. Computer Music Conf., Berlin, Germany, Aug. 2000, pp. 82-85.

[7] D. T. Murphy and D. M. Howard, "2-D digital waveguide mesh topologies in room acoustics modeling," in Proc. COST G-6 Conf. Digital Audio Effects, Verona, Italy, Dec. 2000, pp. 211-216.

[8] L. Savioja, T. Rinne, and T. Takala, "Simulation of room acoustics with a 3-D finite difference mesh," in Proc. Int. Computer Music Conf., Aarhus, Denmark, Sep. 1994, pp. 463-466.

[9] L. Savioja and V. Välimäki, "Reducing the dispersion error in the digital waveguide mesh using interpolation and frequency-warping techniques," IEEE Trans. Speech Audio Process., vol. 8, no. 2, pp. 184-194, Mar. 2000.

[10] S. Van Duyne and J. O. Smith, "The tetrahedral digital waveguide mesh," in Proc. IEEE Workshop Applicat. Signal Process. Audio Acoust., New Paltz, NY, Oct. 1995.

[11] D. E. Dudgeon and R. M. Mersereau, Multidimensional Digital Signal Processing. Englewood Cliffs, NJ: Prentice-Hall, 1984.

[12] J. C. Strikwerda, Finite Difference Schemes and Partial Differential Equations. Pacific Grove, CA: Wadsworth and Brooks, 1989. 